\title{
Optical, Magnetic and Adsorptive Properties of Prepared Copper(II) Oxide
}

\author{
Md. Ataur Rahman, Tajmeri S A Islam and Md. Mufazzal Hossain" \\ Department of Chemistry, University of Dhaka, Dhaka-1000, Bangladesh
}

(Received : 20 February 2019; Accepted : 7 January 2020)

\begin{abstract}
We report the surface morphology, structural propertiesand surface characterization of copper(II) oxide by scanning electron microscope, energy dispersive X-ray spectroscopy, Fourier transformation infrared spectroscopy, attenuated total reflection infrared spectroscopy and X-ray diffraction. All the data of analysis were consistent with each other and bear the evidence for the purity of the prepared oxide. Kubelka-Munk (K-M) theory is used for the determination of direct band gap of the prepared oxide from UV-visible reflectance spectrum. The band gap value of the bulk material was found to be $4.15 \pm 0.05$ $\mathrm{eV}$ from $\mathrm{F}(\mathrm{R})$ versus energy and $(\mathrm{F}(\mathrm{R}) h v)^{\mathrm{n}}$ versus energy plots, with $\mathrm{n}=1 / 2$ and $\mathrm{n}=2$. Molar magnetic susceptibility and effective magnetic moment of the oxide are $3.393 \times 10^{-4}(\mathrm{cgs})$ and $0.895 \mathrm{BM}$ respectively which suggest that the oxide is paramagnetic. Catalytic activity was investigated by the study of adsorption of brilliant red (BR) on the surface of prepared oxide in aqueous medium at room temperature. Adsorption isotherm shows that BR moleculesare adsorbed layer by layer on the surface of oxide. Study of the adsorption phenomenon in this work suggest that copper(II) oxide has significant adsorptive and catalytic property.
\end{abstract}

Keywords: Thermal decomposition, optical property, magnetic property, adsorptive property.

\section{Introduction}

Recent findings reveal some peculiar features of materials especially, metal oxides and semiconductor metal oxide and super conductor metal oxide that particle size, shape and crystal structure play crucial role in their optical, magnetic and electrical properties and antibacterial activities. ${ }^{1,2,3,4}$ Whenever the structure goes to 2D nano-sheet or graphene like shape then these properties change tremendously and photocatalytic activity increase many folds due to larger surface area. Presence of mesopores and wide open geometry ensure larger effective surface area and the shortest pathway for the charge carriers. The surface of nano- $\mathrm{CuO}$ might exhibit special chemical and physical properties that totally vary from the bulk materials. The size and morphology of $\mathrm{CuO}$ has great impact on optical absorption. Many researchers put their attention tosynthesize $\mathrm{CuO}$ due to unique tunable optical properties, magnetic properties ${ }^{4}$ and application in solar cells ${ }^{5}$, gas sensors $^{6}$ and lithium ion batteries ${ }^{7,8}$ of $\mathrm{CuO}$.

Photocatalytic property depends largely on morphology and optical property. Optical property such as band gap drives materials to act as photocatalyst in different portion of electromagnetic radiation. Semiconductor property of material is largely influenced by the band gap. There are two types of transition from one band to another i.e. direct transition and indirection transition. Both of these transitions happen in all semiconductor materials. There is a great paucity of data of direct or indirect band gap energy of $\mathrm{CuO}$. Experimentally the value of band gap was estimated to be in the range of $1.4-1.5 \mathrm{eV} .^{9,10}$ The direct band gap of bulk $\mathrm{CuO}$ is reported to be $3.3 \pm 0.05 \mathrm{eV}$ which depends on morphology and particle size. ${ }^{11}$ Few methods for the determination of the band gap of semiconductor solid material are reported. Among these, UV-visible diffuse reflectance spectroscopy is the most reliable and this method give information about electronic structure of the materials. The reflectance data is treated using Kubelka-
Munk (K-M) theory to get the value of direct band gap. ${ }^{12}$ This theory is widely used for the calculation of band gap of materials and the value obtained is promisingly consistent with the theoretical value.

Solid surfaces have particular importance in industries. Solid surfaces or surface reactions play a vital role in most important areas such as sensors, corrosion, electronic device technology, energy conversion, heterogeneous catalysis and etc. ${ }^{13}$ Among these, heterogeneous catalysis is one of the most economically important areas. It is found that $90 \%$ of the world's industrial materials such as plastics and fertilizer are produced from reactions on heterogeneous catalysts. Prerequisite of surface reactions is the binding or adsorption of substance or reactant at the surface, either by forming chemical bonds or by weak van der Waals interactions. So, catalytic activity of a substance is mainly depends on the adsorptive capacity of the substance. If a substance have high adsorptive capacity then it's catalytic activity is also high and the surface of the substance become more reactive to heterogeneous catalysis.

Rosendo Lopez ${ }^{12}$ have synthesized $\mathrm{TiO}_{2}$ by using sol-gel method and measured the band gap of the prepared and some commercial $\mathrm{TiO}_{2}$ from UV-visible reflectance spectrum using K-M method. Yang $\mathrm{Lu}^{14}$ synthesized 2D nanofilm of $\mathrm{CuO}$ using electrochemical method and studied the photocatalytic properties of oxide using methylene blue and $30 \% \mathrm{H}_{2} \mathrm{O}_{2}$ solution under the irradiation of visible light of $500 \mathrm{~W}$ xenon lamp equipped with wave filter $(\lambda>400 \mathrm{~nm})$.

Magnetic properties of $\mathrm{CuO}$ play an important role in affecting the other properties. As the most of the properties of $\mathrm{CuO}$ depend on size and morphology, the different types of $\mathrm{CuO}$ have been synthesized. ${ }^{15,16,17}$ Our present endeavor is to synthesize $\mathrm{CuO}$ in a very cost effective thermal method. Measurement of the magnetic property, optical property using (K-M) theory and investigation of catalytic property of the oxide were performed. 


\section{Experimental}

\section{Materials}

All the chemicals used for the preparation of sample and in the way to its applications were analytical grade. The chemicals were used without further treatment. Copper(II) carbonate $\left(\mathrm{CuCO}_{3}\right.$, Merck, Germany) was used for the preparation of the sample and brilliant red dye was used for the study of catalytic property of the prepared sample. The deionized water was used throughout the study.

One of the simplest method of preparation of $\mathrm{CuO}$ is thermal decomposition of $\mathrm{CuCO}_{3}$ at it's decomposition temperature $\left(290^{\circ} \mathrm{C}\right) .5 .51 \mathrm{~g} \mathrm{CuCO}_{3}$ was taken in a dry, empty porcelain dish. The sample was spread on the wall of the porcelain dish in thin film. Then, the dish was put in a muffle furnace (ISUZU seisakushoCo.,Ltd) and calcined. The temperature of the furnace was maintained in the range of $295^{\circ} \mathrm{C}-300^{\circ} \mathrm{C}$. The decomposition of $\mathrm{CuCO}_{3}$ was carried out for two hours.

\section{Characterization of the prepared oxide}

Morphology, purity and composition of the prepared oxide were investigated using scanning electron microscopy (JEOL, JSM-6490LA,SEM), energy dispersive X-ray spectroscopy (EDS) coupled with SEM. The crystalline structureof the prepared oxide was analyzed by X-ray diffraction (Philips PW 1830, XRD) using $\mathrm{Cu}-\mathrm{K} \alpha_{1}$ radiation $(\lambda=1.5406 \AA)$. Fourier transform infrared (FTIR) and attenuated total reflection (ATR) spectra were recorded at room temperature in wavenumber of $400-600 \mathrm{~cm}^{-1}$ in a FTIR spectrometer. Reflectance of the sample was measured using UV Spectrophotometer (UV-1800, SHIMADZU) to determine the band gap energy of the sample.

\section{Magnetic property}

Magnetic susceptibility of the prepared sample was measured in a magnetic susceptibility balance (MK1, Sherwood Scientific Ltd) using $\mathrm{MgCl}_{2}$ as standard at $20^{\circ} \mathrm{C}$.

\section{Optical property}

The band gap energy $\left(E_{g}\right)$ of the prepared sample was determined by measuring the reflection spectrum (reflectance as a function of wavelength) in UV-visible spectrophotometer. K-M method which is used to determine the $\mathrm{E}_{\mathrm{g}}$ is based on the following equation

$$
F(R)=\frac{(1-R)^{2}}{2 R}
$$

Where $\mathrm{R}$ is reflectance; $\mathrm{F}(\mathrm{R})$ is proportional to the excitation coefficient $(\alpha)$. A modified K-M function is obtained by multiplying the $F(R)$ function by hv, using the corresponding coefficient (n) associated with an electronic transition. By plotting $(\mathrm{F}(\mathrm{R}) \times \mathrm{h} v)^{\mathrm{n}}$ as a function of energy in $\mathrm{eV}, \mathrm{E}_{\mathrm{g}}$ of semiconductor particles can also be determined. The equation

$$
\alpha(\mathrm{h} v) \approx \mathrm{B}\left(\mathrm{h} v-\mathrm{E}_{\mathrm{g}}\right)^{\mathrm{n}}
$$

is considered. Where $n=2$ for an indirect allowed transition (plotted as $\alpha(\mathrm{hv})^{2}$ versus $\mathrm{E}$ ); $\mathrm{n}=1 / 2$ for a direct allowed transition (plotted as $\alpha(\mathrm{h} v)^{1 / 2}$ versus $\left.\mathrm{E}\right)^{18}$. Both K-M and modified K-M function is used to calculate $\mathrm{E}_{\mathrm{g}}$ of the prepared oxide and these are compared with each other.

\section{Adsorptive property}

Catalytic activity of the prepared oxide was investigated by studying the adsorption of $\mathrm{BR}$ on the oxide at room temperature. Equilibrium time for the adsorption of BR on the oxide was found to be an hour. Adsorption was studied using different initial concentrations of $\mathrm{BR}$, providing the other experimental conditions same. 50.0 mL BR solutions of different initial concentrations were taken in the different bottles and $0.05 \mathrm{~g}$ of oxide sample was added in each bottle at a time. Then, the bottles were put in the shaker (LSB18, Grant Instruments Ltd) and were shaken for an hour. The solutions were then collected in different semi-micro test tubes and centrifuged for 20 minutes in a centrifuge machine (Pro-Analytical C2006, Centurion Scientific). The centrifugate was analyzed using UV spectrophotometer at the $\lambda_{\max }$ of BR. Amount of BR adsorbed on oxide was calculated using the following equation.

$$
q_{t}=\left(C_{i}-C_{t}\right) \times \frac{V M}{W} \times 1000
$$

Where, $C_{i}$ and $C_{t}$ are the initial concentration and concentration at time of $\mathrm{BR}$ in $\mathrm{molL}^{-1}, \mathrm{~V}$ is the volume $(\mathrm{L})$ of solution, $\mathrm{M}$ is the molecular weight of $\mathrm{BR}\left(\mathrm{gmol}^{-1}\right)$ and $\mathrm{W}$ is the amount of $\mathrm{CuO}(\mathrm{g})$

\section{Results and Discussion}

\section{Characterization of the prepared oxide}

SEM images (Fig. 1) of the oxide show that sample is composed of small particles with different geometrical shapessuch as cubic, tetragonal, hexagonal and rod like shape. The particles are of different size and these particles coagulate and agglomerate to form a lump of spherical shape. The surface of the individual particle is homogeneous and smooth. But the surface of the coagulated particles in the form of lump is heterogeneous and the arrangement makes lots of pores and cavities on the surface. These indicate that the prepared oxide may have better adsorptive property.

The EDX spectrum of the sample (Fig. 2) shows that the sample is composed of only $\mathrm{Cu}$ and $\mathrm{O}$. The quantitative analysis shows that $\mathrm{Cu}$ and $\mathrm{O}$ are in a 1:1 stoichiometric ratio since the percent of abundance of the atoms are 50.8 and 49.2 respectively. The results clearly indicate that the prepared oxide is in the form of $\mathrm{CuO}$.

The XRD pattern of the prepared oxide shows a series of characteristics peaks (Fig. 3). All the XRD peaks can be indexed as monoclinic crystal system of $\mathrm{CuO}$ (JCPDS card no. 48-1548) with space group C2/c. Copper(II) oxide produced characteristic peaks indicated by $2 \theta=32.34$, 
$35.41,38.65,48.65,53.41,58.17,66.09,67.93$ and 74.93 as observed by their Millerindices (110), (002), (200), (-202), (020),(202),(022),(220), (004) respectively. ${ }^{19-21}$ No other reflections were detected. These results indicate that the sample contains no impurities. The strong and sharp peaks indicate the high degree of crystallinity of the prepared oxide.
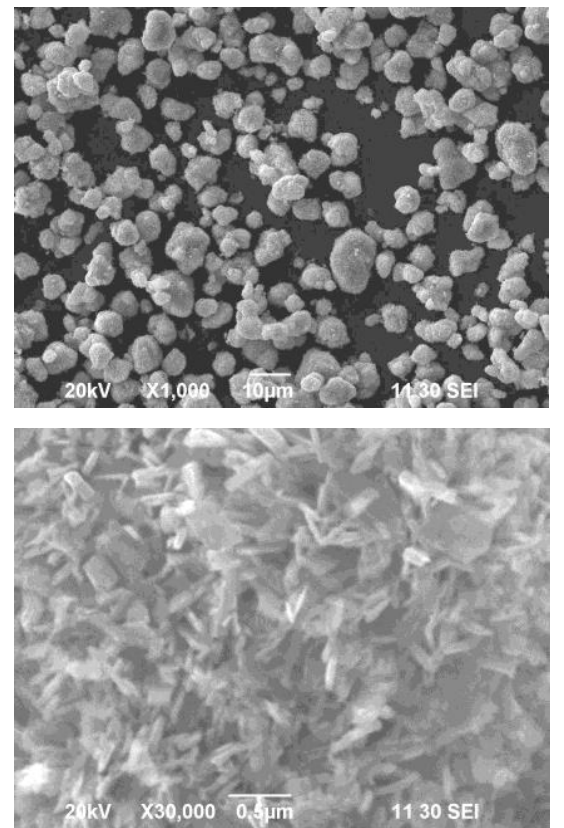

Fig. 1. SEM images of the prepared oxide at different magnifications

The ATR IR spectrum of copper(II) oxide was recorded in the range of 400-600 $\mathrm{cm}^{-1}$ (Fig. 4). The vibrational modes at $403.13,408.82,420.49,423.38,430.13,450.39$, and 458.10 $\mathrm{cm}^{-1}$ are the characteristics peaks of $\mathrm{CuO} .^{22-25}$ Strong peaks at higher frequencies such as $482.2 \mathrm{~cm}^{-1}$ and $478.3 \mathrm{~cm}^{-1}$ appear for the symmetric and asymmetric vibration modes of $\mathrm{Cu}-\mathrm{O}$ group. The weak peaks appears at lower frequencies may be attributed to the wagging vibration of Cu-O group. ${ }^{24}$

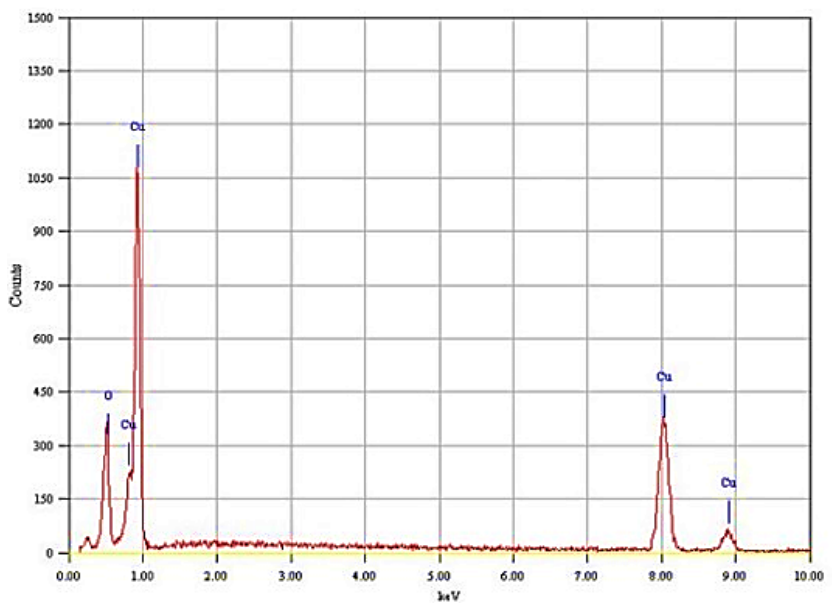

Fig. 2. EDX spectrum of the prepared oxide

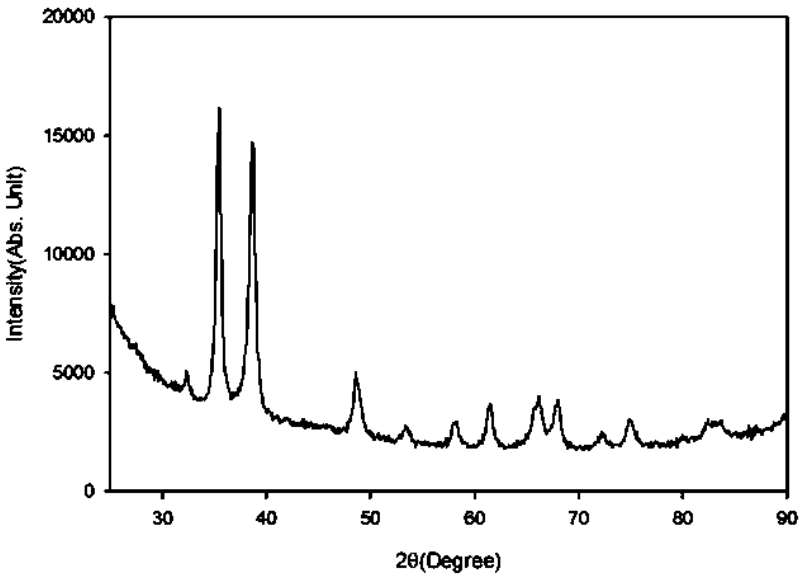

Fig. 3. XRD pattern of the prepared oxide

\section{Optical property}

Band gap energy calculated using K-M and modified K-M functionfrom reflectance spectrum is represented in Figs. 5, 6 and Table 1. The reflectance versus wavelength graph of the prepared oxide represents a breakd ownin the spectrum at about $295 \mathrm{~nm}$ which corresponds to $4.20 \mathrm{eV}$. This should be the band gap of the oxide. K-M graph shows that the band gap is slightly smaller for $n=1 / 2$ than the band gap for $n=2$ (Table 1). There result agrees well with result presented in the Fig. 5. The values of band gap for the bulk material calculated using different methods are precise and more accurate. All these methods indicate that direct allowed inter band transition occurs at $4.2 \pm 0.05 \mathrm{eV}$, which agree with the value calculated for copper(II) oxide sample of different source ${ }^{11}$. Band gap energies of $\mathrm{SnO}_{2}, \mathrm{TiO}_{2}$ and $\mathrm{ZnO}$ are $3.5 \mathrm{eV}, 3 \mathrm{eV}$ and $3.2 \mathrm{eV}$ respectively. Usually the metal oxides which have band gap in the range of 2.2 to 3.5 $\mathrm{eV}$ behave as semiconductor metal oxide and they show efficient photocatalytic activity. ${ }^{25}$ Band gap of $\mathrm{CuO}$ is out of this range and this leads to its inefficiency as semiconductor metal oxide and photocatalyst.

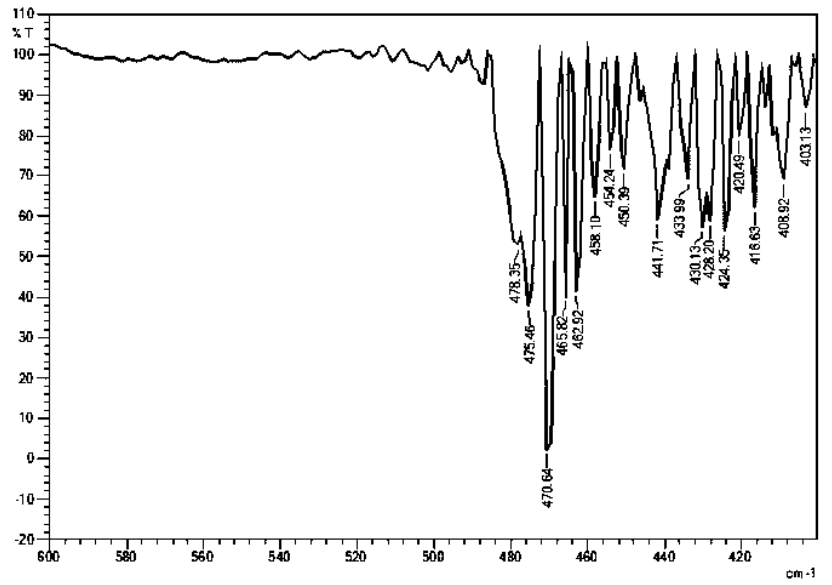

Fig. 4. ATR IR spectrum of the prepared oxide 


\section{Magnetic property}

Magnetic susceptibility of the oxide was measured using $\mathrm{MnCl}_{2}$ as standard at $293 \mathrm{~K}$. Any positive value of the susceptibility indicates that the material is paramagnetic and zero or negative value indicates that it is diamagnetic. Paramagnetic substance is attracted by the magnetic field in the presence of an external magnetic field. The origin of the magnetic property is the unpaired electron in the atomic or molecular orbital. Mass susceptibility, molar susceptibility and effective magnetic moment of the prepared oxide are given in Table 2. All of these values are positive and indicate that the oxide is paramagnetic. The $\mathrm{Cu}^{2+}$ ion contains $\mathrm{d}^{9}$ electronic configuration with an unpaired electron and this unpaired electron contributes to the magnetic property of the oxide. On the other hand, $\mathrm{Cu}^{+}$has $3 \mathrm{~d}$ orbital which is fully occupied and have no unpaired electron, so become diamagnetic. Therefore, $\mathrm{Cu}^{+}$ion is absent in the sample.

\section{Adsorptive property}

Adsorption of a substance is surface phenomenon. Adsorption of adsorbate on adsorbent depends on surface morphology, surface charge of the adsorbent and also the type of the adsorbent materials. Another factor that plays an important role on the adsorptive property is adsorbentadsorbate interactioni. e. solute-solvent interaction. If the interaction is weak and decay vary rapidly with distance, then it usually leads to monolayer adsorption and the succeeding layers diminish in the bulk phase. Other type of adsorption is multilayer adsorption, in which interfacial layer or region persist over several molecular dimensions due to slower decay of interactions. The majority of adsorption isotherm maybe grouped in the six types. $^{26,27}$

Table 1. Band gap energy of copper(II) oxide using different methods

\begin{tabular}{|c|l|c|}
\hline Sample & \multicolumn{1}{|c|}{ Method } & $\begin{array}{c}\text { Calculated } \\
\text { Band gap }(\mathrm{eV})\end{array}$ \\
\hline \multirow{4}{*}{$\begin{array}{c}\text { Copper(II) } \\
\text { oxide }\end{array}$} & $\begin{array}{l}\text { Reflectance versus wavelength } \\
(\mathrm{nm})\end{array}$ & 4.20 \\
\cline { 2 - 3 } & $\mathrm{F}(\mathrm{R})$ versus Energy, E (eV) & 4.20 \\
\cline { 2 - 3 } & $(\mathrm{F}(\mathrm{R}) \mathrm{h} v)^{1 / 2}$ versus Energy, E (eV) & 4.15 \\
\cline { 2 - 3 } & $(\mathrm{F}(\mathrm{R}) \mathrm{h} v)^{2}$ versus Energy, E (eV) & 4.25 \\
\hline
\end{tabular}

Table 2. Data for the magnetic properties of copper(II) oxide

\begin{tabular}{|c|c|c|c|c|c|}
\hline $\begin{array}{c}\text { Temp. } \\
(\mathrm{K})\end{array}$ & $\mathrm{R}-\mathrm{R}_{\mathrm{o}}$ & $\begin{array}{c}\text { Mass } \\
\text { susceptibility } \\
\text { (CGS) }\end{array}$ & $\begin{array}{c}\text { Molar } \\
\text { susceptibility } \\
\text { (CGS) }\end{array}$ & $\begin{array}{c}\text { Effective } \\
\text { magnetic } \\
\text { moment } \\
(\mathrm{BM})\end{array}$ & $\begin{array}{c}\text { Magnetic } \\
\text { Property }\end{array}$ \\
\hline 295 & 396 & $4.265 \times 10^{-6}$ & $3.393 \times 10^{-4}$ & 0.895 & Paramagnetic \\
\hline
\end{tabular}
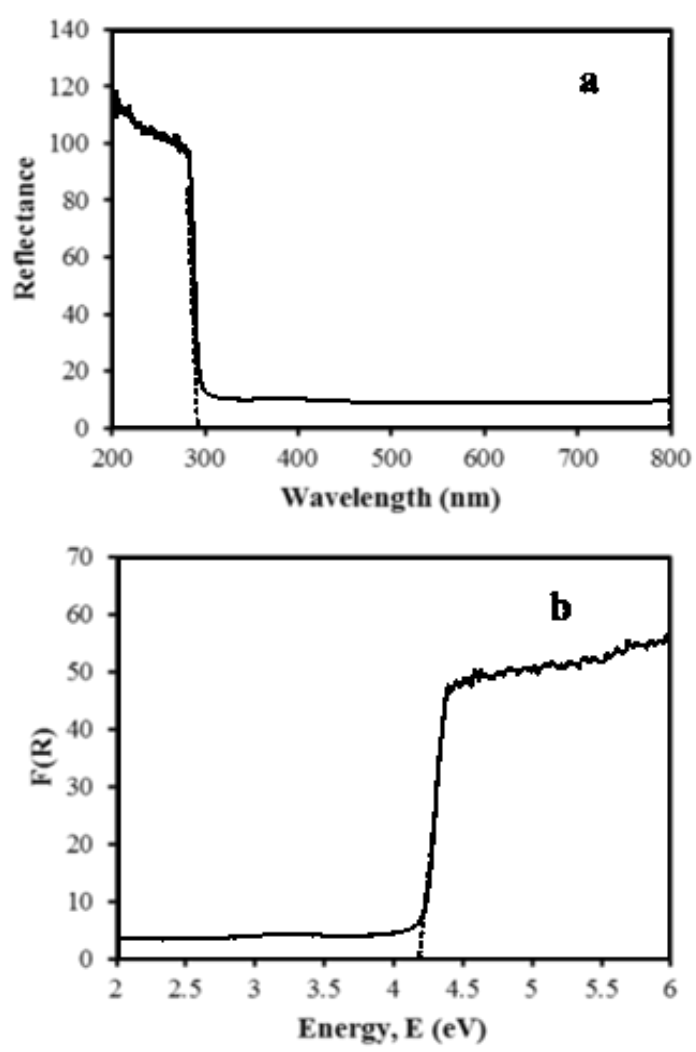

Fig. 5. Graphical representation of reflectance and $F(R)$ spectra for prepared oxide without considering electronic transitions: $\mathbf{a}$. reflectance versus wavelength and $\mathbf{b}$. $\mathrm{F}(\mathrm{R})$ versus energy
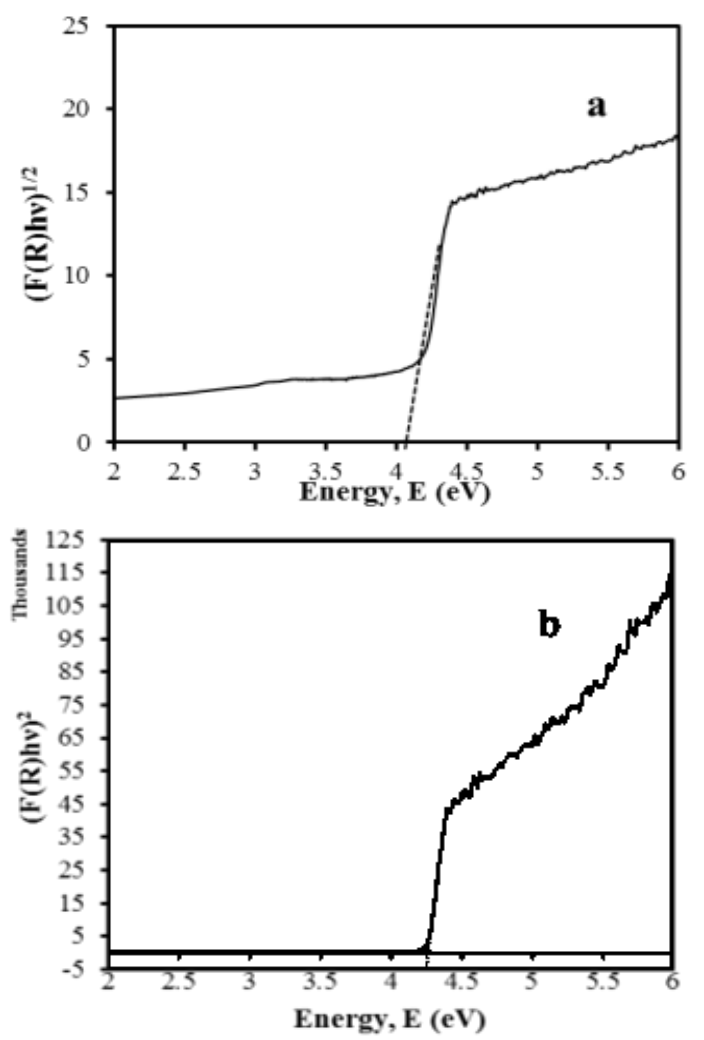

Fig. 6. Graphical representation of modified Kubelka-Munk theory for the prepared oxide: a. $(\mathrm{F}(\mathrm{R}) \mathrm{h} v)^{1 / 2}$ versus energy and b. $(\mathrm{F}(\mathrm{R}) \mathrm{h} v)^{2}$ versus energy 

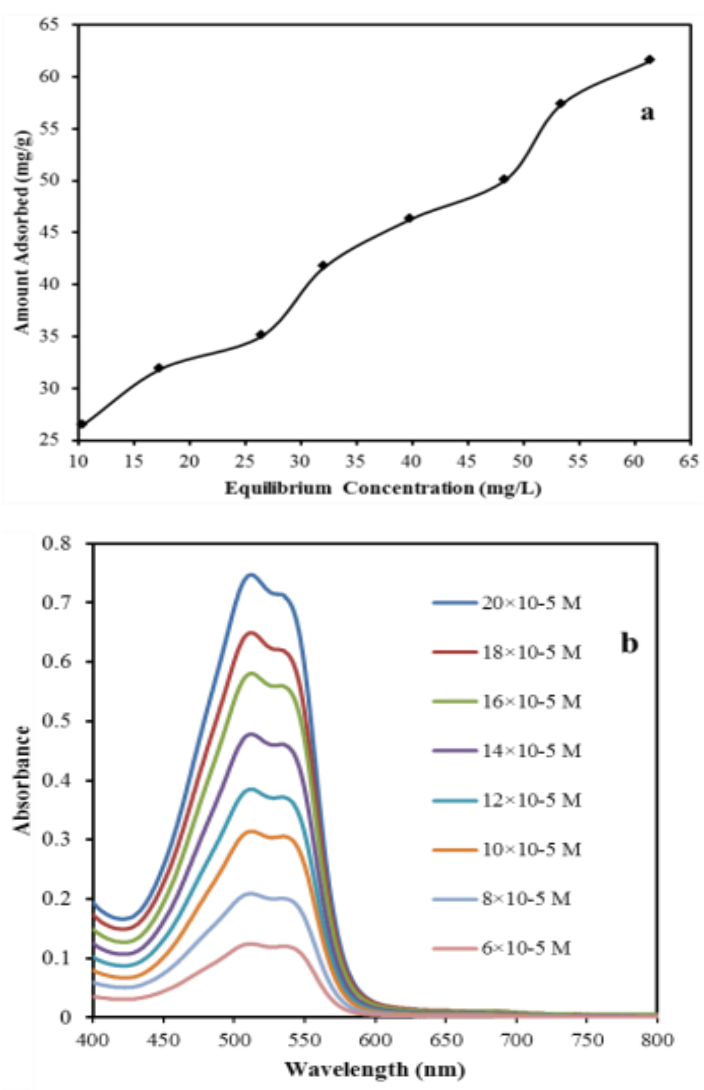

Fig. 7. a. Adsorption isotherm for the adsorption of $B R$ on prepared $\mathrm{CuO}$ at $25^{\circ} \mathrm{C}$, b. Spectrum of BR after adsorption for different initial concentration of $\mathrm{BR}$

Adsorption isotherm for the adsorption of BR on the surface of copper(II) oxide is shown in Fig. 7(a). Our system follows the type VI isotherm, one of the six type of isotherms. This type also describes the adsorption of argon or krypton on graphitised carbon blacks at liquid nitrogen temperature. ${ }^{27}$ According to the model, BR molecules areadsorbed layer by layer on the surface of the oxide and thus form multilayer. The step-height signifies the monolayer capacity for each adsorbed layer and remains almost constant for several adsorbed layers. The arrangement of prepared oxide particles leads to follow this adsorption isotherm type and also persists the influence of interaction between oxide surface and BR molecules to some greater extent. The particles arrange in such a way that the surface of the agglomerates becomes porous (Fig. 1).The diameter of the pore seems to be greater than the $B R$ molecules. BR molecules can easily enter into the pores and seem to be trapped by the oxide particles. These molecules give rise to the formation of a monolayer. After this, the surface also remains unoccupied that leads to the formation of another layer. The interaction of adsorbent and BR is considerably strong to persist this influence to some extent. This interaction also plays crucial role in the formation of multilayer. When the adsorbateare adsorbed in the multilayer on the adsorbent surface, the amount of adsorbate obviously is greater. The net result is that the adsorptive property of the adsorbent increases. Amount adsorbed at equilibrium time is $61.58 \mathrm{mg} / \mathrm{g}$ when initial concentration of dye is $20 \times 10^{-5} \mathrm{M}$. These results indicate the efficient adsorptive activity of the copper(II) oxide.

\section{Conclusion}

Copper(II) oxide prepared from thermal decomposition of copper(II) carbonate was characterized using several techniques. The results from different techniques are consistent with each other. The XRD results indicate that the oxide is a pure copper(II) oxide does not contain any copper(I) oxide. Kubelka-Munk (K-M) theory has been used to measure the direct band gap which was found to be $4.15 \pm 0.05 \mathrm{eV}$ from $\mathrm{F}(\mathrm{R})$ versus energy and $(\mathrm{F}(\mathrm{R}) \mathrm{h} v)^{\mathrm{n}}$ versus energy plots, with $n=1 / 2$ and $n=2$. Molar magnetic susceptibility and effective magnetic moment of the oxide are $3.393 \times 10^{-4}(\mathrm{cgs})$ and $0.895 \mathrm{BM}$ respectively. These results suggest that the oxide is paramagnetic. Adsorption phenomenon of this oxide found in this work suggests the multilayer formation with a high efficiency of adsorption.

\section{References}

1. Ananth, A., S. Dharaneedharan, M. S. Heo,Y. S. Mok, 2015. Copper Oxide Nanomaterials: Synthesis, Characterization and Structure-Specific Antibacterial Performance. Chem. Eng. J. 262, 179-188.

2. Rackauskas, S., A. G. Nasibulin, H. Jiang, 2009. A Novel Method for Metal Oxide Nanowire Synthesis. Nanotechnology. 20(16), 165603.

3. Rehman, S., A. Mumtaz, S. K. H., 2011 Size Effects on the Magnetic and Optical Properties of $\mathrm{CuO}$ Nanoparticles. $J$. Nanoparticle Res. 13 (6), 2497-2507.

4. Kastner, M. A., R. J. Birgeneau,G. Shirane,Y. Endoh, 1990. Magnetic, Transport, and Optical Properties of Monolayer Copper Oxides. Rev. Mod. Phys. 70 (3), 897-928.

5. Liu, Y.,L. Liao, J. Li, C. Pan, 2007. From Copper Nanocrystalline to $\mathrm{CuO}$ Nanoneedle Array: Synthesis, Growth Mechanism, and Properties. J. Phys. Chem. C. 111 (13), 5050-5056.

6. Lu, D. L., J. H., R. W., J. G., 2010. Conductometric Chemical Sensor Based on Individual $\mathrm{CuO}$ Nanowires. Nanotechnology. 21 (48), 485502.

7. Ben-Moshe, T.,I. Dror, B. Berkowitz, 2009. Oxidation of Organic Pollutants in Aqueous Solutions by Nanosized Copper Oxide Catalysts. Appl. Catal. B Environ. 85 (3), 207211.

8. Wang, C., D. Higgins,F. Wang, D. Li,R. Liu,G. Xia,N. Li,G. $\mathrm{Wu}, 2014$. Controlled Synthesis of Micro/nanostructured $\mathrm{CuO}$ Anodes for Lithium-Ion Batteries. Nano Energy. 9, 334-344.

9. Wang, Y.,S. Lany, J. Ghanbaja,Y. FagotRevurat,Y. P. Chen, F. Soldera, D. Horwat, F. Mücklich, J. F. Pierson, 2016. Electronic Structures of $\mathrm{Cu}_{2} \mathrm{O}, \mathrm{Cu}_{4} \mathrm{O}_{3}$, and $\mathrm{CuO}$ : A Joint Experimental and Theoretical Study. Phys. Rev. B. 94 (24), 245418.

10. Ghijsen, J.,L. H. Tjeng, J. van Elp, H. Eskes, J. Westerink, G. A. Sawatzky, M. T. Czyzyk, 1988. Electronic Structure of $\mathrm{Cu}_{2} \mathrm{O}$ and CuO. Phys. Rev. B. 38 (16), 11322-11330. 
11. Koffyberg, F. P.,F. A. Benko,1982. A Photoelectrochemical Determination of the Position of the Conduction and Valence Band Edges of P-type CuO. J. Appl. Phys. 53(2), 1173-1177.

12. Gómez, R. L., 2012. Band-Gap Energy Estimation from Diffuse Reflectance Measurements on Sol-gel and Commercial $\mathrm{TiO}_{2}$ : A Comparative Study. J. Sol-Gel Sci. Technol. 61 (1), 1-7.

13. McCash, E. M. Surface Chemistry; Oxford University Press, 2001.

14. Lu, Y.,X. Liu, K. Qiu, J. Cheng, W. Wang,H. Yan,C. Tang,J. K. Kim,Y. Luo, 2015. Facile Synthesis of Graphene-Like Copper Oxide Nanofilms with Enhanced Electrochemical and Photocatalytic Properties in Energy and Environmental Applications. ACS Appl. Mater. Interfaces. 7 (18), 96829690.

15. Lu, Y.,K. Qiu, D. Zhang, J. Lin, J. Xu,X. Liu,C. Tang,J. K. Kim, Y. Luo, 2014. Cost-Effective CuO Nanotube Electrodes for Energy Storage and Non-Enzymatic Glucose Detection. RSC Adv. 4 (87), 46814-46822.

16. Banerjee, A.,U. Singh, V. Aravindan, M. Srinivasan,S. Ogale, 2013. Synthesis of $\mathrm{CuO}$ Nanostructures from $\mathrm{Cu}$ Based Metal Organic Framework (MOF-199) for Application as Anode for Li-Ion Batteries. Nano Energy. 2 (6), 11581163 .

17. Sun, Y.,Y. Xia, 2002. Shape-Controlled Synthesis of Gold and Silver Nanoparticles. Science. 298 (5601), 2176 LP2179.

18. Nguyen, M. A.,N. M. Bedford, Y. Ren, E. M. Zahran,R. C. Goodin,F. F. Chagani, L. G. Bachas, M. R. Knecht, 2015. Direct Synthetic Control over the Size, Composition, and Photocatalytic Activity of Octahedral Copper Oxide Materials: Correlation Between Surface Structure and Catalytic Functionality. ACS Appl. Mater. Interfaces. 7 (24), $13238-13250$.

19. Munawar, K.,M. A. Mansoor, W. J. Basirun, M. Misran, N. M. Huang, M. Mazhar, 2017. Single Step Fabrication of
CuO-MnO-2 $\mathrm{TiO}_{2}$ Composite Thin Films with Improved Photoelectrochemical Response. RSC Adv.7 (26), 1588515893.

20. Sadollahkhani, A., Z. Hussain Ibupoto, S. Elhag, O. Nur,M. Willander,2014. Photocatalytic Properties of Different Morphologies of $\mathrm{CuO}$ for the Degradation of Congo Red Organic Dye. Ceram. Int. 40 (7 PART B), 11311-11317.

21. Ghaedi, M., A. M. Ghaedi,M. Hossainpour, A. Ansari, M. H. Habibi, A. R. Asghari,2014. Least Square-Support Vector (LS-SVM) Method for Modeling of Methylene Blue Dye Adsorption Using Copper Oxide Loaded on Activated Carbon: Kinetic and Isotherm Study. J. Ind. Eng. Chem. 20 (4), 1641-1649.

22. Kliche, G.,Z. V. Popovic,1990. Far-Infrared Spectroscopic Investigations on CuO. Phys. Rev. B. 42 (16), 10060-10066.

23. Senthil Kumar, P., M. Selvakumar, S. G. Babu, S. K. Jaganathan, S. Karuthapandian, S. Chattopadhyay, 2015. Novel $\mathrm{CuO} /$ chitosan Nanocomposite Thin Film: Facile HandPicking Recoverable, Efficient and Reusable Heterogeneous Photocatalyst. RSC Adv. 5 (71), 57493-57501.

24. Munawar, K.,M. A. Mansoor,W. J. Basirun, M. Misran, N. M. Huang,M. Mazhar,2017. Single Step Fabrication of CuO$\mathrm{MnO}_{2}-\mathrm{TiO}_{2}$ Composite Thin Films with Improved Photoelectrochemical Response. RSC Adv. 7 (26), 1588515893.

25. Akira Fujishima, $\mathrm{TiO}_{2}$ Photocatalysis Fundamentals and Applications, BKC, Inc. 2001

26. Weidenthaler, C., 2011. Pitfalls in the Characterization of Nanoporous and Nanosized Materials. Nanoscale. 3 (3), 792810.

27. Sing, K. S., D. H. Everett, R. A. Haul, L. Mouscou, R. A. Pierotti, J. R., T. S., 1985. Reporting Physisorption Data for Gas/solid Systems with Special Reference to the Determination of Surface Area and Porosity (Recommendations 1984). Pure Appl. Chem. 57 (4), 603-619. 